\title{
Propiedades psicométricas de la Drive for Muscularity Scale (DMS) en una muestra de hombres y mujeres jóvenes chilenos
}

\author{
Neli Escandón-Nagel ${ }^{1}$, María José Baeza-Rivera ${ }^{1}$, Josefa Larenas-Said ${ }^{1}$ y Esteban Caamaño-Mardones ${ }^{1}$ \\ ${ }^{1}$ Universidad Católica de Temuco, Temuco, Chile.
}

Psychometric properties of the Drive for Muscularity Scale (DMS)

in a sample of young Chilean men and women

\begin{abstract}
Drive for muscularity, although it mainly occurs in men, is also experienced by women. It is important to have instruments to measure this construct, which is the reason why the aim of this study was to evaluate the psychometric properties of the Drive for Muscularity Scale (DMS) in Chilean adolescents and young adults $(n=602)$. The DMS and the SATAQ-4 were administered. The confirmatory factor analysis showed two factors, one representing attitudes and the other behaviors, in addition to a second-order factor. This structure had a good fit for both sexes, although for women it was necessary to remove two items. Regarding sex, men showed higher scores and, with respect to age, adolescents manifested greater drive for muscularity. For both sexes a positive correlation between the DMS and the athletic/body ideal internalization scale was observed. These results confirm that the Chilean version of the DMS has adequate psychometric properties.
\end{abstract}

Keywords: Drive for muscularity; body image; adolescence; validation.

Resumen: La obsesión por la musculatura, si bien suele presentarse mayormente en hombres, también la experimentan mujeres. Es importante contar con instrumentos para medir este constructo, por lo que el objetivo de este estudio fue evaluar las propiedades psicométricas del Drive for Muscularity Scale (DMS) en una muestra chilena de adolescentes y jóvenes $(n=602)$. Se administró el DMS y el SATAQ-4. El análisis factorial confirmatorio arrojó dos factores, uno de actitudes y otro de conductas, además de un factor de segundo orden. Esta estructura tuvo un buen ajuste para ambos sexos, aunque en las mujeres fue necesario eliminar dos ítems. En cuanto al sexo, los hombres presentan mayor puntuación y, respecto a la edad, los adolescentes manifiestan mayor obsesión. Se observó una correlación positiva entre DMS y la escala de internalización del ideal atlético/ corporal. Estos resultados constatan que la versión chilena del DMS presenta adecuadas propiedades psicométricas.

Palabras clave: Obsesión por la musculatura; imagen corporal; adolescencia; validación.

\section{Introducción}

Investigaciones previas sobre la imagen corporal se han centrado, generalmente, en la preocupación por alcanzar una imagen corporal delgada en mujeres, lo que

Recibido: 19 de abril 2020; aceptado: 25 de febrero 2021

Correspondencia: Neli Escandón-Nagel, Departamento de Psicología, Facultad de Ciencias de la Salud, Universidad Católica de Temuco, Manuel Montt 056, 4780000, Temuco, Chile. Correo-e: nescandon@uct.cl. Agradecimientos: esta investigación fue financiada por el proyecto interno de la Universidad Católica de Temuco, Chile, PRO FONDECYT 2017PF-NE-03. ha llevado a concluir que los hombres, en comparación con las mujeres, estarían menos insatisfechos con sus cuerpos (Cruzat-Mandich etal., 2016; Durán et al., 2013; González-Carrascosa et al., 2013). Sin embargo, existen estudios que han evidenciado el interés masculino por el logro de un cuerpo más voluminoso y musculado, lo que da cuenta de que también experimentan insatisfacción respecto a su imagen corporal (Aylwin et al., 2016; Behar y Arancibia, 2015; Grieve, 2007).

Por otra parte, en el último tiempo ha habido un cambio en la evaluación de la imagen corporal en ambos sexos (Barrientos-Martínez et al., 2014; Kelley et al., 
2010). Particularmente, en el caso de las mujeres, estas presentan una mayor preocupación que antes por el aumento de su masa muscular, lo cual podría derivar en una mayor obsesión por la musculatura, dejando de ser algo exclusivo de los hombres, siendo entonces necesario prestar mayor atención a este fenómeno, tanto en hombres como en mujeres (Barrientos-Martínez et al., 2014; Hernández-Martínez et al., 2017).

La obsesión por la musculatura se ha definido como la motivación por lograr un cuerpo musculado ideal, junto al grado de preocupación que un individuo puede presentar respecto al aumento de su masa corporal (Rodríguez, 2013; McCreary, 2007; McCreary y Sasse, 2000). Esta obsesión por la musculatura puede llevar al desarrollo de Dismorfia Muscular (DM), descrito como una alteración de la imagen corporal en que se subestima, tanto el tamaño corporal, como la fuerza física, lo que lleva al desarrollo de conductas compensatorias desadaptativas (Parent, 2013; Pope et al., 1997).

Si bien la obsesión por la musculatura se presenta en ambos sexos, suele ser más marcada en hombres, tal como constatan estudios como el de McCreary et al. (2004) y Kyrejto et al. (2008), aunque estos últimos autores añaden que las diferencias por sexo disminuyen cuando la preocupación se focaliza en la tonificación muscular, en lugar del tamaño de los músculos. Las diferencias previamente descritas han suscitado interés por generar más estudios respecto a la obsesión por la musculatura tanto en hombres como en mujeres, a fin de comprender las particularidades que este fenómeno puede adquirir en cada sexo (McCreary et al., 2004).

Se ha observado que la DM y la obsesión por la musculatura se manifiestan cuando las personas perciben presiones sociales para ajustarse a un determinado ideal corporal, que en este caso corresponde a un cuerpo fuerte y musculoso (Compte y Sepúlveda, 2014; Didie et al., 2010). Desde una perspectiva del ciclo vital, ésta tendería a manifestarse principalmente en la adolescencia y adultez joven, etapas en las que más presiones sociales se reciben respecto al logro de un ideal corporal, siendo las personas más vulnerables a ellas que en otras fases de la vida (McCabe y Riciardelli, 2003; Saffon y Saldarriaga, 2014).

Específicamente, en Chile se ha observado que el inicio de sintomatología asociada a algún Trastorno de la Conducta Alimentaria (TCA) e imagen corporal, como puede ser la obsesión por la musculatura, se suele dar entre los 14 y 25 años, es decir, en la adolescencia o adultez joven (Cruzat-Mandich et al., 2014), evidenciándose, además, que tan solo un $16,8 \%$ de los jóvenes se encuentra satisfecho con su apariencia (Cruzat-Mandich et al., 2016).

Al comparar a adolescentes con adultos jóvenes chilenos se logra evidenciar que son los adolescentes quienes muestran mayor insatisfacción corporal y más presiones respecto a la imagen corporal (Cruzat-Mandich et al., 2016). Lo anterior coincide con la literatura, puesto que la adolescencia se constituye en una etapa de autoconocimiento y vinculación con el entorno fuertemente marcada por la autoimagen y mediada por las experiencias tempranas y las influencias culturales promovidas en los medios de comunicación (Behar et al., 2016; Raich, 2013).

Por todo lo anterior, es importante contar con instrumentos que permitan medir este fenómeno. Entre las escalas existentes se encuentra la Muscle Appearance Satisfaction Scale (MASS) de Mayville et al. (2002), que evalúa los dominios cognitivos, conductuales y afectivos de la DM, el cual se ha adaptado en Latinoamérica a población tanto peruana como mexicana masculina, presentando adecuadas propiedades psicométricas (Cookdel Aguila et al., 2016; López-Cuautle et al., 2013).

Por otro lado, está el Muscle Dysmorphia Disorder Inventory (MDDI) (Hildebrandt et al., 2004) para evaluar DM, el que en Latinoamérica fue validado en población argentina masculina (Compte et al., 2019).

A pesar de que los instrumentos señalados poseen adecuadas propiedades psicométricas y son de fácil uso e interpretación, estos se han enfocado en medir la DM. Una escala que se desarrolló para evaluar la obsesión por la musculatura es Drive for Muscularity Scale de McCreary y Sasse (2000), la cual consta de 15 ítems tipo Likert (1: nunca; 6: siempre) de fácil y breve administración. A través de un análisis factorial exploratorio, en hombres $(n=276)$ y mujeres $(n=354)$ escolares y universitarios, McCreary et al. (2004) identificaron dos factores, mostrando niveles adecuados de consistencia interna para cada escala y para la puntuación total.

El primer factor mide las actitudes respecto a la imagen corporal orientadas a la musculatura, mientras que el segundo factor refiere a las conductas orientadas a la musculatura y, además, el instrumento está conformado por un factor global (McCreary et al., 2004). Según lo reportado, la validez convergente de la escala demostró una asociación positiva con la frecuencia del ejercicio, depresión y riesgo de algún Trastorno de la Conducta Alimentaria (TCA), y una asociación negativa con autoestima (McCreary y Sasse, 2000).

McCreary et al. (2004) obtuvieron resultados distintos entre hombres y mujeres, específicamente en las estrategias empleadas para obtener una imagen corporal musculada y, además, encontraron diferencias entre ambos sexos para lograr sus ideales físicos a través del entrenamiento físico. Los mismos autores recomendaron excluir lo referido a la ingesta de esteroides anabólicos, pero otros estudios han corroborado su inclusión (McPherson et al., 2010; Sepulveda et al., 2016). 
En América Latina existe evidencia de su uso en población masculina brasileña, mexicana y argentina, corroborando sus adecuadas propiedades psicométricas en las tres poblaciones (Campana et al., 2013; Compte et al., 2015; Escoto et al., 2013). En población mexicana se identifican tres factores; incorporándose un factor vinculado a la adherencia hacia el entrenamiento, el que incorpora la realización de ejercicio físico y el consumo de suplementos, sin embargo, presentó baja consistencia interna (Escoto et al., 2013). Los resultados en población brasileña consideraron ambos factores originales de la escala, encontrándose correlación entre el ejercicio físico y los factores del DMS, como también asociaciones entre una imagen corporal centrada en la musculación y los hábitos respecto al ejercicio físico (Campana et al., 2013). En la población argentina se consideraron los factores originales de la escala y se encontraron asociaciones positivas con variables relacionadas a la insatisfacción corporal, como también el ejercicio físico respecto a los factores (Compte et al., 2015).

A partir de lo mencionado previamente, al ser escasos los estudios en Latinoamérica y al no tener medidas previas validadas en Chile que determinen la obsesión por la musculatura en una población joven, tanto en hombres como en mujeres, se hace evidente la necesidad de disponer de una versión chilena de la escala DMS que permita evaluar adecuadamente este constructo. Sobre todo, si se tiene en cuenta que la obsesión por la musculatura se ha asociado a diversos comportamientos compulsivos; ejercicios excesivos, ingesta de alimentos excesiva o insuficiente, alteración en la calidad de vida, depresión clínica, intentos de suicidio, uso de sustancias (como los esteroides anabólicos) y deterioro del funcionamiento social y ocupacional (Cunningham et al., 2017).

Por lo tanto, este estudio tiene como objetivo evaluar las propiedades psicométricas del DMS en una muestra de adolescentes y adultos jóvenes chilenos de ambos sexos. Particularmente, se busca evaluar la confiabilidad de la escala y su validez de constructo, convergente y de criterio.

De acuerdo con los antecedentes previamente expuestos, se espera obtener adecuadas propiedades psicométricas, mayor obsesión de la musculatura en hombres que en mujeres, así como también mayor obsesión por la musculatura en adolescentes que en adultos jóvenes.

\section{Método}

\section{Diseño y participantes}

Se utilizó un diseño no experimental, transversal. Por medio de un muestreo no probabilístico por convenien- cia, se accedió a una muestra total de 602 participantes, adolescentes y jóvenes, de entre 15 y 29 años de edad ( $M$ $=20.15 ; D T=3.06)$ de la ciudad de Temuco, Chile. De ellos, el $46.2 \%$ son hombres $(n=278)$, en tanto que el $53,8 \%$ son mujeres $(n=324)$. Se dividió a la muestra en subgrupos según edad, por una parte, adolescentes de 15 a 17 años $(n=147,24.4 \%)$ y, por otro lado, adultos jóvenes de 18 a $29(n=455,75.6 \%)$.

Respecto al índice de masa corporal (IMC), la media de la muestra cae en la categoría de normopeso $(M=$ $24.58 ; D T=4.76) \mathrm{y}$, en relación a la actividad física, el $45.2 \%(n=271)$ declara realizar algún tipo de actividad física a lo menos una vez a la semana, mientras que el $54.8 \%(n=331)$ informa no realizar actividad física.

En la Tabla 1 se presenta información referida al nivel socioeconómico de los participantes, de acuerdo con la escala ESOMAR (Adimark, 2000).

Tabla 1. Distribución de la muestra según nivel socioeconómico

\begin{tabular}{lc}
\hline & Nivel socioeconómico \\
\hline Bajo & $5.68 \%$ \\
Medio bajo & $21.86 \%$ \\
Medio & $32.87 \%$ \\
Medio alto & $29.09 \%$ \\
Alto & $10.50 \%$ \\
\hline
\end{tabular}

\section{Instrumentos}

Escala de Obsesión por la Musculatura, Drive for Muscularity Scale (DMS, McCreary y Sasse, 2000). Instrumento autoadministrado compuesto por 15 ítems, en el cual las personas deben contestar en un formato tipo Likert la frecuencia de actitudes y conductas orientadas a la obsesión por la musculatura. Las alternativas de respuesta van desde 1 (nunca) a 6 (siempre). Un ejemplo de ítem es «Desearía ser más musculoso (a)». En el estudio original de McCreary y Sasse (2000) se encontraron buenas propiedades psicométricas, con un alfa de Cronbach de .84. Recientemente este instrumento fue validado en España por Sepúlveda, Parks, Pellegrin, Anastasiadou, y Blanco (2016), confirmándose sus buenas propiedades psicométricas. Esta versión fue la que se utilizó de base para su adaptación a Chile mostrando en el presente estudio un alpha de Cronbach de 0.86 en mujeres y 0.88 en hombres.

Cuestionario de Actitudes Socioculturales hacia la Apariencia-4, Sociocultural Attitudes Towards Appearance Questionnaire-4 (SATAQ-4, Schaefer et al., 2015). Se 
basa en el «Tripartite influence model» (Van den Berg et al., 2002), el cual resalta la influencia de los medios de comunicación, de los pares y de la familia en la insatisfacción corporal y en el desarrollo de TCA. Consta de 22 ítems en escala tipo Likert de 5 puntos (desde $1=$ completamente en desacuerdo; a $5=$ completamente de acuerdo) y está conformado por 5 sub-escalas que evalúan influencias socioculturales: 3 escalas de presión social (presión de la familia, presión de los pares y presión de los medios para alcanzar la apariencia física ideal) y 2 de internalización de ideales corporales (internalización del ideal de delgadez e internalización del ideal atlético/ muscular). En el presente estudio se utilizó solo la escala de internalización del ideal atlético/corporal, en que un ejemplo de ítem es «Es importante para mi parecer atlético/a». El alfa de Cronbach para esta subescala fue de .88 . Este instrumento se encuentra validado en Chile por los autores de este artículo, evidenciando adecuadas propiedades psicométricas y confirmándose su estructura factorial original (manuscrito en preparación).

Además de estos instrumentos, se recogieron datos sociodemográficos para caracterizar a la muestra, como edad, sexo y nivel socioeconómico. También se recogió información de peso y talla por autoinforme, a fin de calcular el IMC, y se consultó sobre la realización de actividad física.

\section{Procedimiento}

Se utilizó como base la traducción española de Sepúlveda et al. (2016), que luego se administró como pilotaje a un grupo de jóvenes $(n=10)$ con características similares a los de la muestra, quienes dieron sugerencias para las adecuaciones del lenguaje a Chile. Además, respecto al instrumento original, se invirtió la escala Likert, que originalmente iba de 1 (siempre) a 6 (nunca); de modo de generar que, a mayor número, mayor frecuencia de la conducta o actitud evaluada, ya que en el pilotaje se detectó que era más sencillo de comprender para los participantes utilizar la escala Likert de 1 (nunca) a 6 (siempre).

Se estableció contacto con distintas universidades y colegios de la ciudad de Temuco, Chile, para acceder a los participantes del estudio. La aplicación de los instrumentos se realizó entre septiembre de 2018 y octubre de 2019 , de manera colectiva, con una duración de aproximadamente 20 minutos, posterior a la firma del consentimiento informado. En el caso de menores de edad, se contó tanto con el consentimiento del apoderado, así como también, con el asentimiento informado del menor. Este estudio fue aprobado por el Comité de Ética de la Universidad Católica de Temuco, Chile.

\section{Análisis estadístico}

Los datos fueron analizados con el software STATA 16 (StataCorp). Se realizaron análisis descriptivos para caracterizar a los participantes del estudio y para comprobar la estructura factorial propuesta por los autores de la escala, se realizó un análisis factorial confirmatorio (AFC) utilizando el método de estimación de máxima verosimilitud, en este análisis se evalúa la bondad de ajuste del modelo teórico a los datos y las cargas factoriales. En este caso se probó una estructura de segundo orden, tanto para hombres como para mujeres. Los indicadores de bondad de ajuste utilizados fueron: chi-cuadrado $(p<.05)$, el Índice de Ajuste Comparativo (Comparative Fit Index (CFI > .95)), el Error de Aproximación Cuadrático Medio [Root Mean Square Error of Approximation (RMSEA < .08], el índice Tucker-Lewis (TLI > .95) y el Promedio de los Residuales Estandarizados [Standardized Root Mean Square Residual $($ SRMR < .05)]. La consistencia interna de la escala fue estimada por medio del coeficiente Alfa de Cronbach.

Además, para estimar validez de criterio, se utilizó ANOVA factorial, a fin de comparar según grupo de edad y sexo. También se probó la validez convergente, por medio de análisis correlacionales entre obsesión por la musculatura y la subescala de internalización del ideal atlético/corporal del SATAQ-4.

\section{Resultados}

\section{Análisis factorial y confiabilidad}

Los resultados se presentan por separado, para hombres y mujeres. En ambos casos no se cumple el supuesto de normalidad multivariada $(p \leq .001)$, por lo que se utilizó la corrección de Satorra-Bentler.

Respecto a las mujeres, en la Figura 1 se observan las cargas factoriales de los indicadores. Para el factor denominado Actitudes, las cargas factoriales fluctúan entre .67 y .83 ; mientras que para el factor de Conducta las cargas factoriales tienden a ser más bajas, desde .34 a .80. Se eliminaron dos reactivos, el 5 («Intento consumir tantas calorías como puedo en un día») y el 10 («Pienso en tomar esteroides anabólicos, por ejemplo: clembuterol, anavar, winstrol, dianabol, etc.»), debido a que las cargas factoriales fueron menores a 0.30 .

Los indicadores de bondad de ajuste fueron: $\chi_{(\mathrm{SB})}^{2}(63)$ $=142.18, p<.001 ; \mathrm{CFI}_{(\mathrm{SB})}=.91 ; \mathrm{TLI}_{(\mathrm{SB})}=.89 ; \mathrm{RM}-$ $\mathrm{SEA}_{(\mathrm{SB})}=.062 ; \mathrm{SRMR}=.069$. La confiabilidad total es de .86 , mientras que la del factor de Actitud es de $.90 \mathrm{y}$ el de Conducta es de .70; todo esto excluyendo los ítems 5 y 10 , ya que como se indicó anteriormente, fue necesario eliminarlos. 


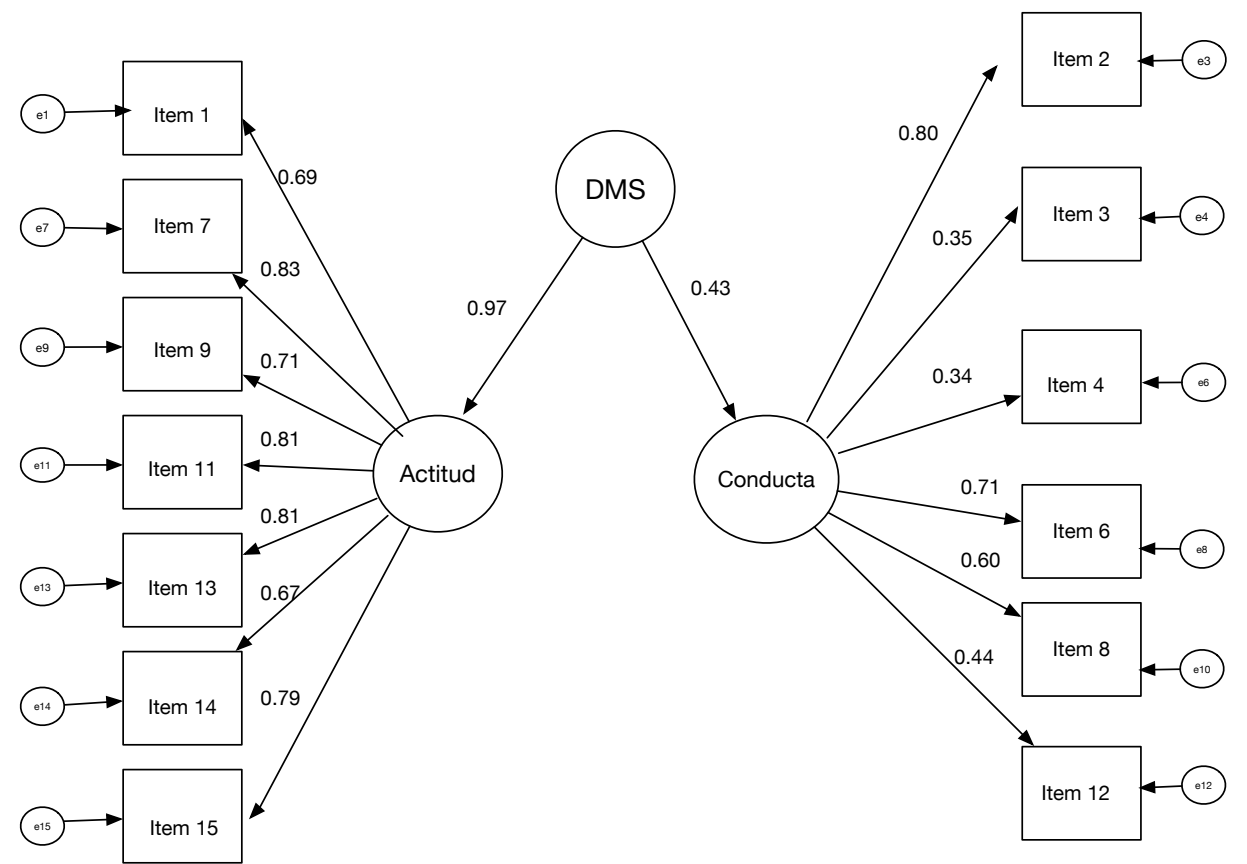

Fuente: Elaboración propia.

Figura 1: Modelo basado en el grupo de mujeres.

En cuanto a los hombres, en la Figura 2 se observan las cargas factoriales de los indicadores. Para el factor Actitudes, las cargas factoriales fluctúan entre .64 y .79; mientras que para el factor de Conducta, las cargas factoriales tienden a ser más bajas desde .49 a .77 .
Los indicadores de bondad de ajuste fueron: $\chi_{(\mathrm{SB})}^{2}(88)$ $=310.182, p \leq .001 ; \mathrm{CFI}_{(\mathrm{SB})}=.84 ; \mathrm{TLI}_{(\mathrm{SB})}=.81 ; \mathrm{RM}-$ $\mathrm{SEA}_{(\mathrm{SB})}=.095 ; \mathrm{SRMR}=.082 . \mathrm{La}$ confiabilidad total es de .88 , mientras que la del factor de Actitud es de $.89 \mathrm{y}$ el de Conducta es de .84 .

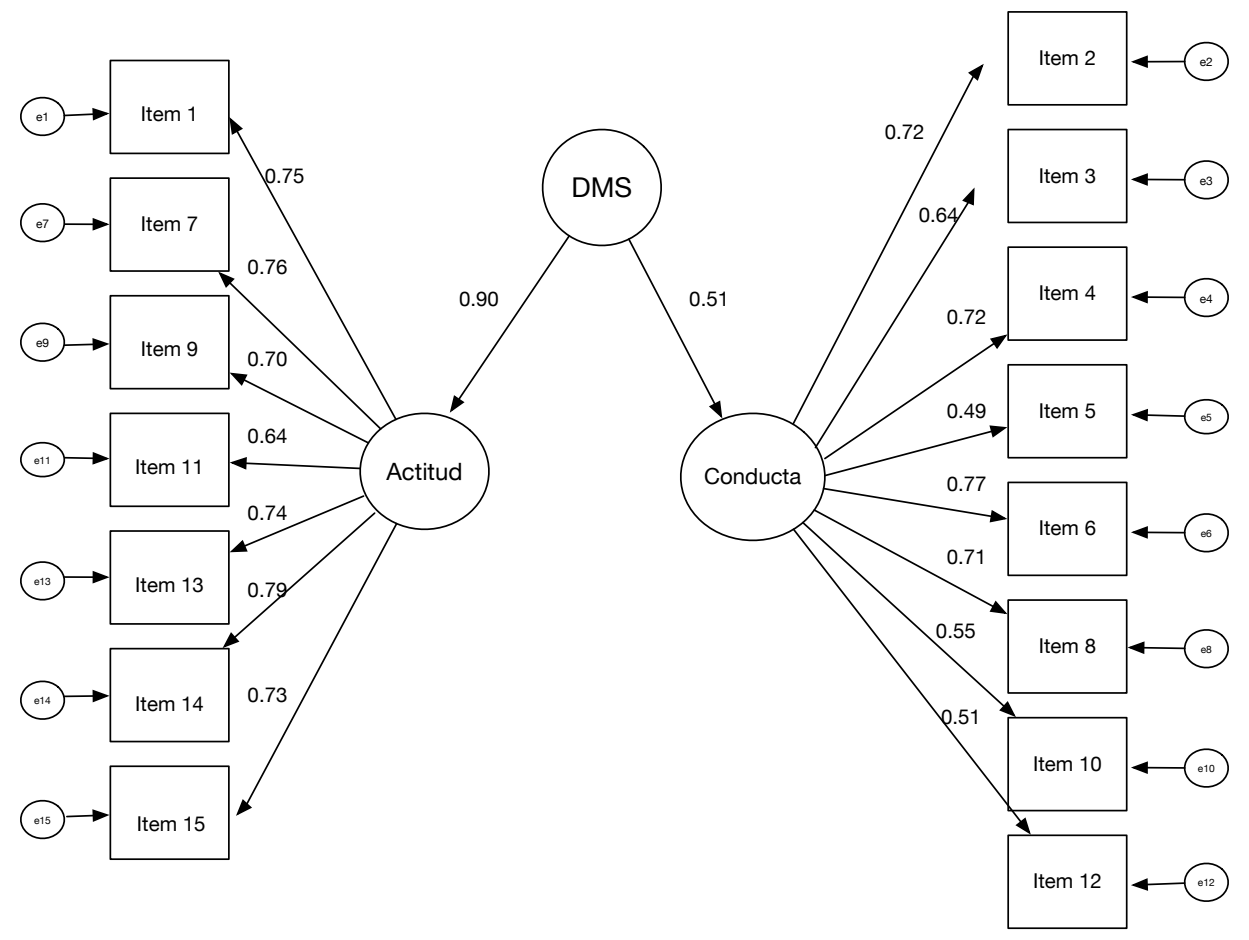

Fuente: Elaboración propia.

Figura 2: Modelo basado en el grupo de hombres. 
En la Tabla 2 se presentan los indicadores de ajuste para los modelos de hombres y mujeres.

Debido a los resultados arrojados por el análisis factorial, particularmente en el caso de mujeres, los si- guientes resultados que se reportan son a partir de la versión de 13 ítems, es decir, excluyendo el ítem 5 y el 10; en tanto que, para los hombres, se consideran los 15 ítems.

Tabla 2. Indicadores de ajuste para los modelos de hombres y mujeres

\begin{tabular}{lcccccc}
\hline & $\chi_{(\mathrm{SB})}^{2}$ & $p$ & $\mathrm{CFI}_{(\mathrm{SB})}$ & TLI $_{(\mathrm{SB})}$ & RMSEA $_{(\mathrm{SB})}$ & SRMR \\
\hline Mujeres & 142.18 & $<.001$ & .91 & .89 & .062 & .069 \\
Hombres & 310.182 & $<.001$ & .84 & .81 & .095 & .082 \\
\hline
\end{tabular}

Fuente. Elaboración propia

Validez convergente y de criterio

Respecto a la validez convergente se identifica una correlación positiva entre la puntuación global del DMS y la sub-escala de internalización del ideal atlético/corporal del SATAQ-4, tanto en hombres $(r=.67, p<.001)$ como en mujeres $(r=.48, p=<.001)$, siendo de mayor magnitud la asociación en el grupo de hombres.

Además, en cuento a la validez de criterio, el ANOVA factorial arrojó, tal como se aprecia en la Tabla 3, que la obsesión por la musculatura se explica por cada uno de los factores por separado, es decir, por sexo y edad $(p<.05)$, con un tamaño del efecto pequeño en el caso de la comparación por grupo etario $\left(\eta^{2}\right.$ parcial $=$ $.01)$ y medio para el factor sexo $\left(\eta^{2}\right.$ parcial $\left.=.06\right)$.

En lo referido a las subescalas del instrumento, se observa que en ambas existen diferencias según sexo con un tamaño del efecto medio $\left(\eta^{2}\right.$ parcial $\left.=.06\right) \mathrm{y}$, particularmente en el caso de la subescala de Conductas, se detectan, además, diferencias dadas por el grupo de edad, pero con un tamaño del efecto pequeño $\left(\eta^{2}\right.$ parcial $\left.=.02\right)$.
Tabla 3. ANOVA factorial

\begin{tabular}{llccc}
\hline & \multicolumn{1}{c}{ Variable } & $F$ & g.l. & $\begin{array}{c}\eta^{2} \\
\text { parcial }\end{array}$ \\
\hline Escala total & Grupo de edad & $4.54^{*}$ & 1 & .01 \\
& Sexo & $39.78^{* *}$ & 1 & .06 \\
& Grupo de edad x sexo & 0.78 & 1 & .01 \\
Actitudes & Grupo de edad & 0.79 & 1 & .00 \\
& Sexo & $38.73^{* *}$ & 1 & .06 \\
& Grupo de edad x sexo & 0.73 & 1 & .00 \\
Conductas & Grupo de edad & $12.35^{* *}$ & 1 & .02 \\
& Sexo & $35.33^{* *}$ & 1 & .06 \\
Hombres & Grupo de edad x sexo & 0.19 & 1 & .00 \\
\hline
\end{tabular}

Nota. $* p<.05 * * p<.01$

Específicamente, y considerando los estadísticos descriptivos que se exponen en la Tabla 4, los hombres presentan mayor obsesión por la musculatura que las mujeres, independientemente del grupo etario al que pertenezcan; a

Tabla 4. Descriptivos del DMS según sexo y edad

\begin{tabular}{lcccc}
\hline & & $\begin{array}{c}\text { Mujeres } \\
(n=324) \\
M(D T)\end{array}$ & $\begin{array}{c}\text { Hombres } \\
(n=278) \\
M(D T)\end{array}$ & $\begin{array}{c}\text { Total } \\
(N=602) \\
M(D T)\end{array}$ \\
\hline $\begin{array}{l}\text { Adolescentes } \\
(n=147)\end{array}$ & Actitudes & $2.08(1.26)$ & $2.85(1.19)$ & $2.43(1.28)$ \\
& Conductas & $1.44(0.64)$ & $1.84(0.92)$ & $1.62(0.81)$ \\
& Escala total & & & \\
$\begin{array}{l}\text { Adultos jóvenes } \\
(n=455)\end{array}$ & Actitudes & $2.08(1.06)$ & $2.66(1.15)$ & $2.35(1.14)$ \\
& Conductas & $1.25(0.46)$ & $1.59(0.74)$ & $1.41(0.63)$ \\
& Escala total & & & $1.88(0.75)$ \\
$(N=602)$ & & $1.70(0.68)$ & $2.09(0.78)$ & $2.37(1.18)$ \\
& Actitudes & $2.08(1.11)$ & $2.71(1.16)$ & $1.46(0.68)$ \\
& Escala total & $1.72(0.72)$ & $2.14(0.82)$ & $1.91(0.79)$ \\
\hline
\end{tabular}


la vez que el grupo de adolescentes manifiesta mayor obsesión por la musculatura que los adultos jóvenes, sin importar si se trata de hombres o mujeres.

\section{Discusión}

El objetivo de la presente investigación fue evaluar las propiedades psicométricas de la escala de obsesión por la musculatura, Drive for Muscularity Scale (DMS), en una muestra de adolescentes y adultos jóvenes chilenos de ambos sexos. Tal como se propuso en las hipótesis, los resultados dan cuenta de un instrumento con adecuadas propiedades psicométricas que es capaz de distinguir que los hombres presentan mayor obsesión por la musculatura que las mujeres y que los adolescentes puntúan más alto que los adultos jóvenes.

Respecto a las propiedades psicométricas, los resultados dan cuenta de la validez de constructo constatada por el AFC, el cual se realizó por separado para hombres y mujeres. De este modo, se identificó la presencia de dos factores en ambos sexos, actitudes y conductas; y se corroboró un factor global, tal como plantea la estructura original propuesta por McCreary et al. (2004), la cual fue confirmada también por estudios posteriores realizados con muestras de hombres (Campana et al., 2013; Compte, 2015; Escoto et al., 2013). Sin embargo, en el caso de los hallazgos de McCreary et al. (2004), para mujeres se detectó un único factor. Lo mismo sucedió en un estudio reciente realizado con mujeres brasileñas a partir de análisis factorial exploratorio (de Carvalho, da Costa Oliveira, Neves, Meireles, y Ferreira, 2019). Esta discrepancia entre la presente investigación y las anteriores pudiera estar dada por factores culturales, como las creencias asociadas a la obsesión por la musculatura, y porque en ninguno de ellos se utilizó AFC para probar el modelo de dos factores.

De los hallazgos por sexo, se aprecia que en mujeres la estructura fue diferente a la de los hombres, pues se eliminaron dos ítems del factor conductual; el 5 «Intento consumir tantas calorías como puedo en un día», y el 10 «Pienso en tomar esteroides anabólicos (por ejemplo: clembuterol, anavar, winstrol, dianabol, etc.)». A partir de esto, se puede suponer que estas conductas en mujeres tienen relación con el aumento de masa corporal, lo que iría en oposición a la apariencia femenina ideal, pues según distintos autores, el objetivo en cuanto a la imagen corporal en mujeres es lograr una apariencia tonificada y delgada (McCreary et al., 2004; Markula, 1995; Mosewich et al., 2009). Esto es diferente al ideal corporal masculino, ya que, si bien ellas desarrollan preocupación por poseer un cuerpo musculado, el interés y las estrategias que emplean las mujeres estarían enfocadas principalmente en disminuir la masa corporal y la grasa a través de la reduc- ción de ingesta calórica y la realización de ejercicio físico (Kyrejto et al., 2008; McCreary et al., 2004).

Estudios internacionales muestran una prevalencia global de por vida del uso de esteroides anabólicos en hombres de $6.4 \%$ y de $1.6 \%$ para mujeres (Sagoe et al., 2014), por lo que esta conducta podría ser promovida socialmente como una práctica primordialmente masculina, donde el hombre busca una musculatura más gruesa, lo que según se ha estudiado, ha sido masivamente divulgado en los medios de comunicación y la sociedad en general (Raich, 2013, Saffon y Saldarriaga, 2014).

Por otra parte, McCreary et al. (2004) observaron que la inclusión del ítem vinculado al uso de esteroides anabólicos no implica una carga significativa en los factores, sin embargo, existen investigaciones que avalan la inclusión del ítem en población masculina (Campana et al., 2013; McPherson et al., 2010), tal como sucede en el presente estudio. En muestra de fisicoculturistas, este ítem presenta mayor significancia al mostrar mayor variabilidad para contribuir a la escala, incluso estableciéndose como posible indicador de riesgo (McCreary et al., 2004).

Respecto a la validez convergente del DMS, en el presente estudio fue confirmada, pues se correlacionó la puntuación total de la escala con la subescala de internalización del ideal atlético/corporal del SATAQ-4. En tanto que, respecto a la validez de criterio, concordando con investigaciones previas (McCabe y Riciardelli, 2003; Smolak y Stein, 2006), en este estudio se obtuvo mayor obsesión por la musculatura en adolescentes y en hombres, tal como era de esperar.

La mayor obsesión por la musculatura observada en hombres en esta investigación coincide con la evidencia en población masculina mexicana, brasileña y argentina (Campana et al., 2013; Compte, 2015; Escoto et al., 2013). La obsesión por la musculatura en hombres se ha relacionado con una mayor insatisfacción corporal, perfeccionismo y preocupaciones constantes en torno a la dieta y el ejercicio físico que podrían implicar el abuso de sustancias y una mayor comorbilidad con trastornos mentales (Kyrejto et al., 2008; Toro-Alfonso et al., 2010; McCreary et al., 2004).

Los hallazgos en este estudio permiten contar con un instrumento para medir la obsesión por la musculatura en adolescentes y adultos jóvenes chilenos, lo que posibilitará la comparación de resultados en diversos contextos internacionales. La utilidad de este instrumento en Chile hará posible que investigadores y profesionales de la salud logren evaluar la existencia de estas actitudes y conductas, favoreciendo la acción preventiva y las intervenciones oportunas respecto a esta problemática.

Una de las ventajas de este estudio es que incorpora a mujeres, lo que permite entonces la evaluación de este 
constructo no solo en hombres, teniendo en cuenta que actualmente el ideal femenino no se basa solamente en la búsqueda de la delgadez, sino que también de un cuerpo musculado (Barrientos-Martínez et al., 2014; Hernández-Martínez et al., 2017).

En cuanto a las limitaciones de esta investigación, es importante mencionar que la validación del instrumento SATAQ-4 en Chile no está actualmente publicada, sin embargo, el trabajo está siendo desarrollado por los autores de esta investigación.

Es necesario en futuros estudios probar el instrumento en otro tipo de poblaciones, como usuarios/as de gimnasio, fisicoculturistas, deportistas y/o personas adultas. Además, aún se debe investigar la asociación de la obsesión por la musculatura con otros constructos relevantes, como la ansiedad social física. Estudios en un futuro deberán explorar a través de test-restest la confiabilidad del instrumento a lo largo del tiempo.

En definitiva, los resultados de este estudio demuestran que el DMS es un instrumento útil para evaluar la obsesión por la musculatura, tanto en hombres como en mujeres, adolescentes y adultos jóvenes. De este modo, la versión chilena del DMS se constituye en una herramienta simple y de fácil uso en el ámbito de la evaluación psicológica.

\section{Conflictos de intereses}

Los autores declaran no tener conflictos de intereses.

\section{Referencias}

Adimark. (2000). El Nivel Socioeconómico ESOMAR, Manual de aplicación. 1-8. Recuperado de http://www.microweb.cl/idm/ documentos/ESOMAR.pdf

Aylwin, J., Díaz-Castrillón, F., Cruzat-Mandich, C., García, A., Behar, R. y Arancibia, M. (2016). Vivencias y significados en torno a la imagen corporal en varones chilenos. Revista Mexicana de Trastornos Alimentarios, 7(2), 125-134. https:// doi.org/10.22201/fesi.20071523e.2016.2.393

Barrientos-Martínez, N., Escoto, C., Bosques L., Enríquez-Ibarra, J. y Juárez-Lugo, C. (2014). Interiorización de ideales estéticos y preocupación corporal en hombres y mujeres usuarios de gimnasio. Revista Mexicana de Trastornos Alimentarios, 5(1) 29-38. https://doi.org/10.1016/S2007-1523(14)70374-X

Behar, R. y Arancibia, M. (2015). Trastornos de la imagen corporal: Anorexia nerviosa versus anorexia inversa (trastorno dismórfico muscular). Revista Mexicana de Trastornos Alimentarios, 6(2), 121-128. https://doi.org/10.1016/j.rmta.2015.10.005

Behar, R., Arancibia, M., Heitzer, C. y Meza, N. (2016). Trastorno dismórfico corporal: aspectos clínicos, dimensiones nosológicas y controversias con la anorexia nerviosa. Revista Médica de Chile, 144(5), 626-633. https://doi.org/10.4067/S003498872016000500011
Campana, A. N. N. B., Tavares, M. da C. G. C. F., Swami, V. \& da Silva, D. (2013). An examination of the psychometric properties of Brazilian Portuguese translations of the Drive for Muscularity Scale, the Swansea Muscularity Attitudes Questionnaire, and the Masculine Body Ideal Distress Scale. Psychology of Men \& Masculinity, 14(4), 376-388. https://doi.org/10.1037/a0030087

Cook-del Aguila, L., Sanchez-Castro, A. E., Yacila, G. A., Reyes, M. A. y Mayta-Tristán, P. (2016). Adaptación cultural de la versión peruana de la Muscle Appearance Satisfaction Scale (MASS) para dismorfia muscular. Revista Colombiana de Psiquiatría, 45(4), 268-269. https://doi.org/10.1016/j.rcp.2016.04.003

Compte, E. J., Nagata, J. M., Sepúlveda, A. R., Rivas, A., Sbdar, L. S., Menga, S., Rica, R., Torrente, F. \& Murray, S. B. (2019). Assessment and validation of a Spanish version of the Muscle Dysmorphia Disorder Inventory in Argentinian men who exercise: Inventario de Dismorfia Muscular. Body Image, 31, 24-34. https://doi.org/10.1016/j.bodyim.2019.08.002

Compte, E. J. y Sepúlveda, A. R. (2014). Dismorfia Muscular: perspectiva histórica y actualización en su diagnóstico, evaluación y tratamiento. Behavioral Psychology, 22(2), 307-326.

Compte, E. J., Sepúlveda, A. R., de Pellegrin, Y. \& Blanco, M. (2015). Confirmatory factor analysis of the Drive for Muscularity Scale-S (DMS-S) and Male Body Attitudes Scale-S (MBAS-S) among male university students in Buenos Aires. Body Image, 14, 13-19. https://doi.org/10.1016/j.bodyim.2015.02.005

Cruzat-Mandich, C., Díaz-Castrillón, F., Aylwin-Navarro, J., García-Troncoso, A., Behar-Astudillo, R. y Arancibia-Meza, M. (2014). Discursos en anorexia y bulimia nerviosa: un estudio cualitativo acerca del vivenciar del trastorno. Revista Mexicana de Trastornos Alimentarios, 5(2), 70-79. https://doi. org/10.22201/fesi.20071523e.2014.2.282

Cruzat-Mandich, C., Díaz-Castrillón, F., Lizana-Calderón, P. y Castro, A. (2016). Comparación por sexo en imagen corporal, síntomas psicopatológicos y conductas alimentarias en jóvenes entre 14 y 25 años. Revista Médica de Chile, 144(6), 743-750. https://doi.org/10.4067/S0034-98872016000600008

Cunningham, M. L., Griffiths, S., Mitchison, D., Mond, J. M., Castle, D. \& Murray, S.B. (2017). Muscle dysmorphia: An overview of clinical features and treatment options. Journal of Cognitive Psychotherapy, 31(4), 255-271. https://doi.org/10.1891/08898391.31.4.255

de Carvalho, P. H. B., da Costa Oliveira, F., Neves, C. M., Meireles, J. F. F. \& Ferreira, M. E. C. (2019). Is the Drive for Muscularity Scale a valid and reliable instrument for young adult women? BodyImage, 29,1-5.https://doi.org/10.1016/j.bodyim.2019.02.001

Didie, E. R., Kuniega-Pietrzak, T. \& Phillips, K. A. (2010). Body image in patients with body dysmorphic disorder: Evaluations of and investment in appearance, health/illness, and fitness. Body Image, 7(1), 66-69.https://doi.org/10.1016/j.bodyim.2009.09.007

Durán, S., Rodríguez, M. D. P., Record, J., Barra, R., Olivares, R., Tapia, A. y Neira, A. M. (2013). Autopercepción de la imagen corporal en estudiantes universitarios de Chile y Panamá. Revista Chilena de Nutrición, 40(1), 26-32. https://doi. org/10.4067/S0717-75182013000100004

González-Carrascosa, R., García-Segovia, P. y Martínez-Monzó, J. (2013). Valoración de la imagen corporal y de los comportamientos alimentarios en universitarios. Revista de Psicopatología y Psicología Clínica, 18(1), 45-59. https://doi.org/10.5944/rppc. vol.18.num.1.2013.12762 
Grieve, F. G. (2007). A conceptual model of factors contributing to the development of muscle dysmorphia. Eating Disorders, 15, 63-80. https://doi.org/10.1080/10640260601044535

Hernandez-Martinez, A., Gonzalez-Marti, I. \& Contreras Jordan, O. R. (2017). Muscular Dysmorphia in different sports: soccer, gymnastics, weightlifting, swimming and triathlon. Revista de Psicología del Deporte, 26(2), 123-130.

Hildebrandt, T., Langenbucher, J. \& Schlundt, D. G. (2004). Muscularity concerns among men: Development of attitudinal and perceptual measures. Body Image, 1, 169-181. https://doi. org/10.1016/j.bodyim.2004.01.001

Kelley, C. C. G., Neufeld, J. M. \& Musher-Eizenman, D. R. (2010). Drive for thinness and drive for muscularity: Opposite ends of the continuum or separate constructs? Body Image, 7(1), 74-77. https://doi.org/10.1016/j.bodyim.2009.09.008

Kyrejto, J. W., Mosewich, A. D., Kowalski, K. C., Mack, D. E. \& Crocker, P. R. (2008). Men's and women's drive for muscularity: Gender differences and cognitive and behavioral correlates. International Journal of Sport and Exercise Psychology, 6(1), 69-84. https://doi.org/10.1080/1612197X.2008.9671855

López-Cuautle, C., Vázquez-Arévalo, R., Ruíz-Martínez, A. O. y Mancilla-Díaz, J. M. (2013). Propiedades psicométricas del instrumento Muscle Appearance Satisfaction Scale (MASS) en hombres mexicanos. Revista Mexicana de Trastornos Alimentarios, 4(2), 79-88. https://doi.org/10.1016/S2007-1523(13)71995-5

Markula, P. (1995). Firm but shapely, fit but sexy, strong but thin: Postmodern aerobicizing female bodies. Sociology of Sport Journal, 15, 109-137. https://doi.org/10.1123/ssj.12.4.424

Mayville, S. B., Williamson, D. A., White, M. A., Netemeyer, R. G. \& Drab, D. L. (2002). Development of the Muscle Appearance Satisfaction Scale: A self-report measure for the assessment of muscle dysmorphia symptoms. Assessment, 9(4), 351-360. https://doi.org/10.1177/1073191102238156

McCabe, M. P. \& Riciardelli, L. A. (2003). Sociocultural influences on body image and body changes among adolescent boys and girls. Journal of Social Psychology, 143, 5-26. https://doi. org/10.1080/00224540309598428

McCreary, D. R. \& Sasse, D. K. (2000). An exploration of the drive for muscularity in adolescent boys and girls. Journal of American College Health, 48, 297-304.

McCreary, D. R., Sasse, D. K., Saucier, D. M. \& Dorsch, K. D. (2004). Measuring the drive for muscularity: factorial validity of the drive for muscularity scale in men and women. Psychology of Men \& Masculinity, 5(1), 49.

McPherson, K. E., McCarthy, P., McCreary, D. R. \& McMillan, S. (2010). Psychometric evaluation of the Drive for Muscularity Scale in a community-based sample of Scottish men participating in an organized sporting event. Body Image, 7(4), 368-371. https://doi.org/10.1016/j.bodyim.2010.06.001

Mosewich, A. D., Vangool, A. B., Kowalski, K. C. \& McHugh, T. L. F. (2009). Exploring women track and field athletes' meanings of muscularity. Journal of Applied Sport Psychology, 21(1), 99-115. https://doi.org/10.1080/10413200802575742

Parent, M. C. (2013). Clinical considerations in etiology, assessment, and treatment of men's muscularity-focused body image disturbance. Psychology of Men \& Masculinity, 14, 88-100. https://doi.org/10.1037/a0025644

Pope, H. G. J., Gruber, A. J., Choi, P., Olivardia, R. \& Phillips, K. A. (1997). Muscle dysmorphia. An underrecognised form of body dysmorphia disorder. Psychosomatics, 38, 548-557. https://doi.org/10.1016/S0033-3182(97)71400-2

Raich, R. M. (2013). Imagen Corporal: Conocer y valorar el propio cuerpo. Ediciones Pirámide.

Rodríguez, T. J. F. (2013). Alteraciones de la imagen corporal. Editorial Síntesis.

Saffon, S. y Saldarriaga, L. M. (2014). La internalización del ideal de delgadez: factor de riesgo de alteraciones de la imagen corporal y los hábitos de alimentación, generado por la influencia de pares. Revista de Psicología Universidad de Antioquia, 6(1), 75-90.

Sagoe, D., Molde, H., Andreassen, C. S., Torsheim, T. \& Pallesen, S. (2014). The global epidemiology of anabolic-androgenic steroid use: a meta-analysis and meta-regression analysis. Annals of epidemiology, 24(5), 383-398. https://doi. org/10.1016/j.annepidem.2014.01.009

Sepulveda, A. R., Parks, M., de Pellegrin, Y., Anastasiadou, D. \& Blanco, M. (2016). Validation of the Spanish version of the Drive for Muscularity Scale (DMS) among males: Confirmatory factor analysis. Eating Behaviors, 21, 116-122. https://doi. org/10.1016/j.eatbeh.2016.01.010

Schaefer, L. M., Burke, N. L., Thompson, J.K., Dedrick, R. F., Heinberg, L. J., Calogero, R. M., Bardone-Cone, A. M., Higgins, M. K., Frederick, D. A., Kelly, M., Anderson, D. A., Schaumberg, K., Nerini, A., Stefanile, C., Dittmar, H., Clark, E., Adams, Z., Macwana, S., Klump, K. L., Swami, V. (2015). Development and validation of the Sociocultural Attitudes Towards Appearance Questionnaire-4 (SATAQ-4). Psychological Assessment, 27(1), 54-67. https://doi.org/10.1037/a0037917

Smolak, L. \& Stein, J. A. (2006). The relationship of drive for muscularity to sociocultural factors, self-esteem, physical attributes gender role, and social comparison in middle school boys. Body Image, 3(2), 121-129. https://doi.org/10.1016/j. bodyim.2006.03.002

Toro-Alfonso, J., Lugo, K. N. y Bracero, N. B. (2010). Cuerpo y masculinidad: Los desórdenes alimentarios en hombres. Interamerican Journal of Psychology, 44(2), 225-234.

Van Den Berg, P., Thompson, J. K., Obremski-Brandon, K. \& Coovert, M. (2002). The tripartite influence model of body image and eating disturbance: A covariance structure modeling investigation testing the mediational role of appearance comparison. Journal of Psychosomatic Research, 53(5), 10071020. https://doi.org/10.1016/S0022-3999(02)00499-3 
Apéndice 1.

DMS

\begin{tabular}{|cccccc|}
\hline $\mathbf{1}$ & $\mathbf{2}$ & $\mathbf{3}$ & $\mathbf{4}$ & $\mathbf{6}$ & $\mathbf{5}$ \\
NUNCA & POCAS VECES & A VECES & A MENUDO & CASI SIEMPRE & SIEMPRE \\
\hline
\end{tabular}

Para responder, lee atentamente cada frase y marca con un círculo el número (uno por cada frase) que mejor represente tu pensamiento:

\begin{tabular}{|c|c|c|c|c|c|c|c|}
\hline 1 & Desearía ser más musculoso(a) & 1 & 2 & 3 & 4 & 5 & 6 \\
\hline 2 & Levanto pesas para desarrollar músculo & 1 & 2 & 3 & 4 & 5 & 6 \\
\hline 3 & $\begin{array}{l}\text { Tomo suplementos energéticos o proteicos (a base de ca- } \\
\text { feína, té verde, arginina, etc.) }\end{array}$ & 1 & 2 & 3 & 4 & 5 & 6 \\
\hline 4 & $\begin{array}{l}\text { Bebo batidos hipercalóricos para aumentar de peso o bati- } \\
\text { dos proteicos (por ejemplo: polvos de proteínas o mez- } \\
\text { clas de alimentos ricos en proteínas como huevo, frutas, } \\
\text { verduras, frutos secos, etc.). }\end{array}$ & 1 & 2 & 3 & 4 & 5 & 6 \\
\hline 5 & Intento consumir tantas calorías como puedo en un día & 1 & 2 & 3 & 4 & 5 & 6 \\
\hline 6 & $\begin{array}{l}\text { Me siento culpable si pierdo una sesión de entrenamiento } \\
\text { con pesas }\end{array}$ & 1 & 2 & 3 & 4 & 5 & 6 \\
\hline 7 & $\begin{array}{l}\text { Creo que me sentiría más seguro(a) si tuviera más masa } \\
\text { muscular }\end{array}$ & 1 & 2 & 3 & 4 & 5 & 6 \\
\hline 8 & $\begin{array}{l}\text { Otras personas creen que entreno con pesas con demasiada } \\
\text { frecuencia }\end{array}$ & 1 & 2 & 3 & 4 & 5 & 6 \\
\hline 9 & $\begin{array}{l}\text { Creo que me vería mejor si aumentara } 5 \text { kilos en masa } \\
\text { muscular }\end{array}$ & 1 & 2 & 3 & 4 & 5 & 6 \\
\hline 10 & $\begin{array}{l}\text { Pienso en tomar esteroides anabólicos (por ejemplo: } \\
\text { clembuterol, anavar, winstrol, dianabol, etc.) }\end{array}$ & 1 & 2 & 3 & 4 & 5 & 6 \\
\hline 11 & $\begin{array}{l}\text { Creo que me sentiría más fuerte si ganara un poco más de } \\
\text { masa muscular }\end{array}$ & 1 & 2 & 3 & 4 & 5 & 6 \\
\hline 12 & $\begin{array}{l}\text { Creo que mi horario de entrenamiento con pesas interfiere } \\
\text { con otros aspectos de mi vida }\end{array}$ & 1 & 2 & 3 & 4 & 5 & 6 \\
\hline 13 & Creo que mis brazos no son suficientemente musculosos & 1 & 2 & 3 & 4 & 5 & 6 \\
\hline 14 & $\begin{array}{l}\text { Creo que mis pectorales } \mathbf{y} / \mathbf{0} \text { pechos no son suficientemente } \\
\text { musculosos }\end{array}$ & 1 & 2 & 3 & 4 & 5 & 6 \\
\hline 15 & Creo que mis piernas no son suficientemente musculosas. & 1 & 2 & 3 & 4 & 5 & 6 \\
\hline
\end{tabular}

* Se marcan en negrita las modificaciones realizadas al instrumento, respecto a la versión original (consistieron en agregar detalles para su mejor comprensión). 